\title{
Stars in the age of micro-arc-second astrometry
}

\author{
Y. Lebreton \\ GEPI, UMR 8111, Observatoire de Paris-Meudon, 92195 Meudon, France \\ email: Yveline.Lebreton obspm.fr
}

\begin{abstract}
The understanding and modelling of the structure and evolution of stars is based on statistical physics as well as on hydrodynamics. Today, a precise identification and proper description of the physical processes at work in stellar interiors are still lacking (one key point being that of transport processes) while comparison of real stars to model predictions, which implies conversions from the theoretical space to the observational one, suffers from uncertainties in model atmospheres. This results in uncertainties on the prediction of stellar properties needed for galactic studies or cosmology (as stellar ages and masses). In the next decade, progress is expected from the theoretical, experimental and observational sides. I illustrate some of the problems we are facing when modelling stars and possible ways toward their solutions. I discuss how future observational ground-based or spatial programs (in particular those dedicated to micro-arc-second astrometry, asteroseismology and interferometry) will provide precise determinations of the stellar parameters and contribute to a better knowledge of stellar interiors and atmospheres in a wide range of stellar masses, chemical composition and evolution stages.
\end{abstract}

Keywords. stars: interiors, stars: evolution, stars: fundamental parameters, stars: oscillations

\section{Stellar internal structure and evolution studies: goals and tools}

Major goals of stellar structure and evolution studies are (i) to characterize and describe the physics of matter in the extreme conditions encountered in stars, and (ii) to determine stellar properties (like age and mass) that trace the history and evolution of galaxies and constrain cosmological models. To achieve these goals, we rely on numerical stellar models based on input physics that integrate the results of recent theoretical studies, numerical simulations and laboratory experiments. The model input and output are chosen and/or validated by comparison with accurate astronomical observations.

Numerical 2D and 3D hydrodynamical simulations of limited regions of stellar interiors and atmospheres are now under reach of computers. They provide valuable constraints and data for the current standard (1D) stellar models: abundances, convection, rotationally induced instabilities and mixing, magnetic fields (see Asplund 2005; Talon 2007; Zahn 2007, for reviews). In parallel, the physics of stellar plasma is studied in the laboratory with (i) fluid experiments (study of turbulence in rotating, magnetic fluids, see e.g. Richard \& Zahn 1999), (ii) particle accelerators (nuclear reaction cross sections) and, (iii) the so-called high energy-density facilities (based on high power lasers or z-pinches) which aim at exploring the high temperature and high density regimes found in stars, brown dwarfs and giant planets to get information on the equation of state (EOS), opacities or thermonuclear reactions (see Remington et al. 2006).

Modern ground-based and spatial telescopes equipped with high quality instrumentation are in use or under development (VLT-VLTI, JWST, etc.). They provide very accurate data which, after treatment, give access to stellar global parameters: luminosity, radius, mass, effective temperature $T_{\text {eff }}$, gravity $\log g$, abundances. On the other hand, seismic data (such as oscillation frequencies or amplitudes) are being obtained 
from velocities (e.g. Bedding \& Kjeldsen 2007) and from photometry by the space missions MOST (Walker et al. 2003) and CoRoT (Michel et al. 2006). In the next decade, valuable observational data are expected. For instance Gaia (ESA 2000; Perryman et al. 2001), to be launched in 2011, will make astrometric measurements, at the micro-arc second level together with photometric and spectroscopic observations of a huge number of stars covering the whole range of stellar masses, compositions and evolution stages while the Kepler mission, to be launched in 2009, will provide the opportunity to make asteroseismic observations on a wide range of stars (Christensen-Dalsgaard et al. 2007).

In the following, I discuss the different aspects of stellar modeling, the problems encountered, and the perspectives.

\section{Stellar models: input parameters and observational constraints}

\subsection{Input physics for stellar models}

Stellar model calculations require a good description of the physical processes at work. Microscopic physics (opacities, EOS, nuclear reaction rates, atomic diffusion) are now rather well described which improves agreement between models and observations. However, difficulties still arise in the modelling of e.g. (i) cold, dense stars (molecular opacities, non-ideal effects in the EOS), (ii) advanced stages of evolution (nuclear reaction rates), and (iii) stars to be modelled very accurately like the Sun (see Sect. 3.1). On the other hand, despite important recent progress, macroscopic processes (convection, transport of chemicals and angular momentum related to differential rotation and the role of magnetic fields and internal gravity waves) are not yet fully understood (see e.g. Talon 2007).

\subsection{Input from observations and from model atmospheres}

Stellar model calculations involve input and constraints derived from observation. Observational data (e.g. magnitudes, colors, spectra, light and velocity curves, astrometric data) must be treated to get stellar parameters (luminosity, mass, radius, abundances, etc.). Model atmospheres are a crucial step in the analysis, for instance, to predict fluxes in different bands, synthetic spectra, or limb-darkening coefficients. They also provide the boundary conditions for the interior model as well as bolometric corrections and colortemperature conversions. Recently, model atmospheres have been improved by bringing in better atomic and molecular data and by applying more sophisticated algorithms. The output of 2-3D MHD simulations, including NLTE effects are being used to derive abundances, $T_{\text {eff }}$ or $\log g$ from high quality spectra (Asplund 2005; Zahn 2007).

Although internal errors in the determination of stellar parameters are becoming quite small for dwarfs, subgiants, and giants of spectral types A to K (see Table 1 in Lebreton 2005), large systematic errors still remain for cool and hot stars. For instance, the systematic errors on metallicity of metal poor dwarfs and cool giants amount to 0.2-0.3 dex, that is ten times more than the typical internal errors, while differences in $T_{\text {eff }}$-scales can reach up to 200-400 K (Gustafsson 2004; Allende Prieto 2006).

Oscillations have been detected in many stars: solar-like, $\delta$ Scuti, $\beta$ Cephei, $\gamma$ Dor, Cepheids, RR Lyrae, SPB, WD, etc. They result from propagation of acoustic pressure or gravity waves, depending on the mass, evolution stage, chemical composition and excitation mechanism. Valuable constraints can be drawn from the frequencies or their combination (see, e.g. Christensen-Dalsgaard 1988; Roxburgh \& Vorontsov 2003, and references therein). 


\section{Modeling calibrators}

Only a few stars for which we have strong or numerous observational constraints are used as calibrators, i.e. they serve to validate the models and learn the physics. What is learned from calibrators can then be applied to stars with incomplete or less accurate observations. This implies extrapolation to compositions, masses or evolution stages not covered by calibrators. Quantities of astrophysical interest such as age, helium content or distance scale can then be derived for large numbers of stars.

\subsection{Solar modeling: AGS05 mixture and seismology}

The solar photospheric abundances have been re-determined recently on the basis of 3D radiative-hydrodynamical model atmospheres including better atomic data and NLTE effects (Asplund 2005). The new mixture, referred to as AGS05, gives a global metallicity and abundances of C, N, O smaller by 30-40\% than those given by the GN93 mixture (Grevesse \& Noels 1993) derived from 1D hydrostatic models. As a result, the interior opacity is reduced and the solar model no more satisfies the helioseismic constraints (e.g., convection zone depth, sound speed profile). This problem is especially acute in the region between $\sim 0.4$ and $\sim 0.7 R_{\odot}$ (upper part of the radiative zone and shear region below the convective zone, i.e. tachocline). Several authors have shown that the present uncertainties in the input physics of the solar model, in particular the opacities or the atomic diffusion velocities, can hardly explain the differences (see e.g. Montalbán et al. 2006). On the other hand, it has been suggested that the neon abundances could be in error by a factor of at least 2, but the problem is still open (Grevesse et al. 2007).

\subsection{Binary system modeling: the RS Cha and $\alpha$ Centauri binary systems}

The modelling of a binary system should reproduce the observed constraints under the assumption that the two stars have the same age and initial composition. This may allow to infer the values of the model unknowns: age, initial helium, and physical parameters such as the mixing-length parameter for convection or overshooting parameter.

The binary system RS Cha is an interesting SB2 eclipsing system whose components are A-type oscillating stars in a PMS evolution stage corresponding to the onset of the earliest reactions of the CNO cycle. The modeling of the system by Alecian et al. $(2007 \mathrm{a}, \mathrm{b})$ in the light of new accurate observations (masses, radii, metallicity) has shown that, to get agreement between the model and observations, carbon and nitrogen must be depleted with respect to their values in the GN93 mixture. The AGS05 mixture fulfills this condition but to assess this result and the values of the system's age and helium abundance derived from the calibration, it is now necessary to further improve the observational data. In particular, the $[\mathrm{Fe} / \mathrm{H}]$ value should be redetermined using $3 \mathrm{D}$ model atmospheres and it would be valuable to get individual abundances of major elements and better seismic data, the present ones being too crude to provide useful constraints.

The binary system $\alpha$ Centauri is the closest and best-known one. The observed global parameters are accurate (in particular the accuracy on the interferometric radii is at $1 \%$ ) and seismic observations of both stars have allowed to measure the frequencies of several low degree p-modes with the accuracy of $\sigma_{\nu} \simeq 0.3-2.0 \mu H z$. Several authors have performed calibrations of the system (e.g., Miglio \& Montalban 2005). They conclude that it is difficult to find a set of parameters that satisfies simultaneously the global and seismic constraints. As a result, the constraints on the physics of models remain loose, age and initial helium abundance are still poorly determined. It would be interesting to better assess the radii and masses by confirming the parallax of the system (different values were obtained from the analysis of Hipparcos data) and to further improve and enlarge the seismic data that are still scarce and coarse. 


\subsection{Modeling stars in open clusters}

Members of stellar clusters can also serve as calibrators. They can be studied under the assumption that they all have the same age and initial composition but different mass. The initial helium abundance and age of a cluster can be derived from comparison of a model isochrone with observations of cluster stars in the color-magnitude diagram (see Fig. 3 in Lebreton 2005). The surface He abundance $Y$ can be derived from the position of the lower main sequence (MS) and the age from the MS turn-off. Such studies require accurate observations (parallax, magnitude, $T_{\text {eff }},[\mathrm{Fe} / \mathrm{H}]$ ). The metallicity uncertainty affects the $Y$ estimate because of the helium-metallicity degeneracy in the $\mathrm{H}-\mathrm{R}$ diagram. Also, uncertainties on $Y$ and on age come from a poor knowledge of input physics, like envelope or core convection, rotational mixing or atomic diffusion (Lebreton et al. 2001).

The observations of binary stars in a cluster can provide additional constraints. For instance, the position of the low-mass, non-evolved stars in the mass-luminosity plane is related to their helium abundance while physics can be constrained if several binaries spanning a large mass range can be observed. Again the accuracy of the parallax and metallicity are crucial. A study of the Hyades (the only cluster where individual distances have been obtained by Hipparcos and masses measured for a few stars) has shown the limitation of the method due to the uncertainties on metallicity and input physics, and the small number of stars with accurate mass determination (Lebreton et al. 2001).

Investigations by e.g. Mazumdar \& Antia (2001); Basu et al. (2004); Piau et al. (2005) have shown that the seismic analysis of different modes of oscillations should help to probe their inner properties such as the outer convection zone depth and helium content or the convective core boundary, and to estimate their mass and age. For instance, in solar-type stars, the higher the helium abundance in the convective envelope, the deeper the depression in the adiabatic index $\Gamma_{1}$ in the region of second helium ionisation. As shown by Basu et al. (2004), the helium abundance in the envelope of low-mass stars could be derived using the signature of this depression in the p-mode frequencies. This would require that low degree p-modes are observed with a frequency accuracy of $0.01 \%$ and that the mass or radius of a star is known independently.

\section{Deriving astrophysical parameters: the example of stellar ages}

In our Galaxy, the ages of A and F stars are crucial inputs for studying the disc while those of old metal poor stars and globular clusters provide valuable constraints for cosmology. The uncertainty on age depends on many factors (precision of the position in the $\mathrm{H}-\mathrm{R}$ diagram, abundances, knowledge of the model input physics such as convection, rotational mixing, atomic diffusion). The case of globular clusters is discussed by Chaboyer (these proceedings). I focus here on the ages of A-F stars.

\subsection{Ages of $A$ and $F$ stars and the size of their mixed cores}

A-F stars have convective cores on the MS and may be fast rotators in the $\delta$ Scuti instability strip. Therefore, to model these stars, we are faced with difficulties in describing the central extra-mixing by core convection overshoot and rotationally induced mixing in the radiative zone. We have to estimate these effects of rotation from photometric data. These processes modify either the stellar models or the position of the observed star in the $\mathrm{H}-\mathrm{R}$ diagram that, in turn, affects the age determination.

The efficiency of mixing in the stellar core determines the quantity of fuel available to a star with a crucial impact on its lifetime. Overshooting of the convective cores produces an extra-mixing. In model calculations, this extra-mixing is usually crudely parameterized with a coefficient $\alpha_{o v}$, the value of which probably depends on mass, composition and 
evolution stage as shown by empirical calibrations based on binaries and the MS width observations (Ribas et al. 2000; Young et al. 2001; Cordier et al. 2002). Rotationally induced mixing can also bring extra fuel to the stellar engine. Recently, progress has been made in modeling the transport of angular momentum and chemicals resulting from differential rotation (see the review by Mathis et al. 2007).

Goupil \& Talon (2002) have calculated models of a typical A-star including either overshooting or rotational mixing and have found that these distinct processes cannot be discriminated in the $\mathrm{H}-\mathrm{R}$ diagram where they have similar signatures. Goupil \& Talon also showed that if the A-star pulsates as a $\delta$ Scuti star then the signature of the mixing process could be seen in the oscillations frequencies, provided enough modes are observed and identified. We estimate that an uncertainty on age ranging from 13 to $24 \%$ results from a poor knowledge of the inner mixing processes (Lebreton et al. 1995). We expect that in the near future, the improvement of the observed $\mathrm{H}-\mathrm{R}$ diagram (in particular, the luminosities from micro-arc-second astrometry) and the availability of precise seismic data will allow us to reduce this uncertainty down to 3-5\% (Lebreton 2005).

\section{Perspectives in the context of micro-arc-second astrometry}

Today very few calibrators are available to probe the physics of stellar interiors. After Hipparcos, $\sim 200$ stars have distances accurate to better than 1\%. Also, the sample of stars with masses and radii measured with the accuracy better than $1 \%$ remains small. Concerning open clusters, Hipparcos provided precise individual distances only for the Hyades, while the binaries have been analysed in the Hyades and Pleiades only. There is no cluster star known with the solar-like oscillations being detected. Concerning A-F stars, Hipparcos determined the distances of $10^{3}$ A-F stars with an accuracy better than $\sim 10 \%$, while the seismic data have been obtained for quite some stars, but they are often coarse.

In the next decade, we expect that observations of stars will increase both in numbers and quality. Missions dedicated to global astrometry like Gaia (ESA 2000; Perryman et al. 2001) and SIM (Unwin et al. 2007) will measure the parallax with accuracy better than 10 micro-arcseconds. In parallel, the measurements of stellar parameters (magnitudes, temperatures, abundances, masses, radii etc.) are expected to be much improved due to the high resolution spectroscopy and interferometry. High-quality seismic data are expected from several missions: CoRoT will reach an accuracy of $0.1 \mu \mathrm{Hz}$ on frequencies for about 50 targets (mainly solar-like oscillators, $\beta$ Cephei and $\delta$ Scuti) while Kepler will enlarge the seismic sample to thousands of stars with a frequency accuracy of $0.1-0.3 \mu \mathrm{Hz}$.

\subsection{Expected returns from Gaia}

Gaia's astrometry will be complemented by photometry and spectroscopy allowing most masses and evolution stages to be precisely documented. The number of calibrators for stellar physics will be drastically increased and homogeneous global parameters will be provided, e.g., magnitudes, masses and abundances. The parallax of $7 \times 10^{5}\left(21 \times 10^{6}\right)$ stars will be measured with accuracies of at least $0.1 \%(1 \%)$ and the mass of stars in 17000 binary systems will be obtained with accuracies better than $1 \%$. About 120 open clusters (up to $1 \mathrm{kpc}$ ) will be brought to a level of precision better than now existing for the Hyades. Parallax measurements, accurate to $0.5 \%$, will be provided for $5 \times 10^{5}$ A stars and $3 \times 10^{6} \mathrm{~F}$ stars. Furthermore, while Hipparcos yielded direct distances with accuracies better than $12 \%$ for only 11 subdwarfs and 2 subgiants, Gaia will provide (i) precise direct distances for very large samples of subdwarfs and for all subgiants up to $3 \mathrm{kpc}$ and (ii) individual distances with an accuracy better than $10 \%$ for stars in $\sim 20$ 
globular clusters. We will have access to very precise H-R diagrams of very large stellar samples with complementary data (as mass, radius, detailed abundances and seismic data) for various subsamples of stars. The interpretation of these data in the light of future improvements on theoretical, numerical and experimental physics will certainly bring further insights in the understanding of stellar interiors and evolution.

\section{References}

Alecian, E., Goupil, M.-J., Lebreton, Y., Dupret, M.-A., \& Catala, C. 2007a, A\& $A, 465,241$ Alecian, E., Lebreton, Y., Goupil, M.-J., Dupret, M.-A., \& Catala, C. 2007b, A\&A, 473, 181 Allende Prieto, C. 2006, in: S. J. Kannappan, S. Redfield, J. E. Kessler-Silacci, M. Landriau, \& N. Drory (eds.), New Horizons in Astronomy: F. N. Bash Symp., ASP Conf. Ser.,352, 105 Asplund, M. 2005, ARAA, 43, 481

Basu, S., Mazumdar, A., Antia, H. M., \& Demarque, P. 2004, MNRAS, 350, 277

Bedding, T. R. \& Kjeldsen, H. 2007, CoAst, 150, 106

Christensen-Dalsgaard, J. 1988, in: J. Christensen-Dalsgaard \& S. Frandsen (eds.), Advances in Helio- and Asteroseismology, IAU Symp. 123, p. 295

Christensen-Dalsgaard, J., Arentoft, T., Brown, T. M., et al. 2007, CoAst, 150, 350

Cordier, D., Lebreton, Y., Goupil, M.-J., et al. 2002, A\& A, 392, 169

ESA. 2000, White-Book, Gaia - Composition, Formation and Evolution of the Galaxy, Concept and Technology Study Report (ESA-SCI(2000)4), 1-381

Goupil, M. J. \& Talon, S. 2002, in: C. Aerts, T. R. Bedding, \& J. Christensen-Dalsgaard (eds.), Radial and Nonradial Pulsations as Probes of Stellar Physics, ASP Conf. Ser., 259, 306

Grevesse, N., Asplund, M., \& Sauval, A. J. 2007, Space Sci. Revs, 105

Grevesse, N. \& Noels, A. 1993, in: N. Prantzos, E. Vangioni-Flam and M. Casse (eds.), Origin and Evolution of the Elements, CUP, p. 14

Gustafsson, B. 2004, in: A. McWilliam \& M. Rauch (eds.), Origin and Evolution of the Elements, CUP, p. 104

Lebreton, Y. 2005, in: C. Turon, K. S. O'Flaherty, \& M. A. C. Perryman (eds.), The ThreeDimensional Universe with Gaia, ESA SP 576, 493

Lebreton, Y., Fernandes, J., \& Lejeune, T. 2001, A\&A, 374, 540

Lebreton, Y., Michel, E., Goupil, M. J., Baglin, A., \& Fernandes, J. 1995, in: E. Hog \& P. K. Seidelmann (eds.), Astronomical and Astrophysical Objectives of Sub-Milliarcsecond Optical Astrometry, IAU Symp. 166, 135

Mathis, S., Eggenberger, P., Decressin, T., et al. 2007, in: C. Straka, Y. Lebreton, M. Monteiro (eds.), Stellar Evolution and Seismic Tools for Asteroseismology, EAS Publi. Ser. 26, 65

Mazumdar, A. \& Antia, H. M. 2001, A\&SA, 377, 192

Michel, E., Baglin, A., Auvergne, M., et al. 2006, in: M. Fridlund, A. Baglin, J. Lochard, \& L. Conroy, (eds.), The CoRoT Mission, ESA SP 1306, 39

Miglio, A. \& Montalbán, J. 2005, A\&A, 441, 615

Montalbán, J., Miglio, A., Theado, S., Noels, A., \& Grevesse, N. 2006, CoAst, 147, 80

Perryman, M. A. C., de Boer, K. S., Gilmore, G., et al. 2001, A\& $A, 369,339$

Piau, L., Ballot, J., \& Turck-Chièze, S. 2005, A\& A, 430, 571

Remington, B. A., Drake, R. P., \& Ryutov, D. D. 2006, Rev. Mod. Phys., 78, 755

Ribas, I., Jordi, C., \& Giménez, Á. 2000, MNRAS, 318, L55

Richard, D. \& Zahn, J.-P. 1999, A\&SA, 347, 734

Roxburgh, I. W. \& Vorontsov, S. V. 2003, A\&A, 411, 215

Talon, S. 2007, ArXiv e-prints, 708

Unwin, S. C., Shao, M., Tanner, A. M., et al. 2007, ArXiv e-prints, 708

Walker, G., Matthews, J., Kuschnig, R., et al. 2003, PASP, 115, 1023

Young, P. A., Mamajek, E. E., Arnett, D., \& Liebert, J. 2001, ApJ, 556, 230

Zahn, J.-P. 2007, in: F. Kupka, I. Roxburgh, \& K. Chan (eds.), Convection in Astrophysics, Proc. IAU Symp. 239, p. 517 